\title{
STATUS OF THE FIRST STAGE OF LINEAR INDUCTION ACCELERATOR SILUND-21
}

\author{
A.A. Fateev, G.V. Dolbilov, I.I. Golubev, I.N. Ivanov, V.V. Kosukhin, N.I. Lebedev, \\ V.A. Petrov, V.N. Razuvakin, V.S. Shvetsov, M.V. Yurkov \\ Joint Institute for Nuclear Research, 141980 Dubna, Moscow Region, Russia
}

In the present paper we report on status of the project of SILUND-21 LIA which is under development at JINR [1]. SILUND-21 will provide electron beams with the energy up to $10 \mathrm{MeV}$, peak current $\sim 1 \mathrm{kA}$ and pulse duration $50-70 \mathrm{~ns}$. SILUND-21 will serve as a base of experimental facility to study microwave electronics, FEL technique and two-beam acceleration. It is assumed also to perform experiments to adopt the FEL bunching technique for generation of the CLIC driving beam.

\section{Introduction}

The SILUND-21 linear induction accelerator will serve as a base of experimental facility to study microwave electronics, FEL technique and two-beam acceleration. We plan also to perform experiments to adopt the FEL bunching technique for generation of the CLIC driving beam [2]. In this case the driving beam is produced in an FEL amplifier constructed on the base of linear induction accelerator (LIA). One of the main problems to realize such a way of the CLIC driving beam generation is that of LIA. To achieve the required parameters, LIA should provide electron beam with the energy about of $10 \mathrm{MeV}$ and peak current about of $1-2 \mathrm{kA}$ at a high repetition rate. We plan also to study the problems of the beam bunching preservation at further acceleration of the driving beam.

Linear induction accelerator SILUND-21 is constructed using the equipment of LUEK-20 accelerator developed for experimental investigations of the collective method of acceleration [3]. It is assumed to upgrade the main pieces of the LUEK-20 equipment using the experience of construction and operation of SILUND, SILUND-II, SILUND-20 and LUEK-20 linear induction accelerators developed at JINR [3] - [7].

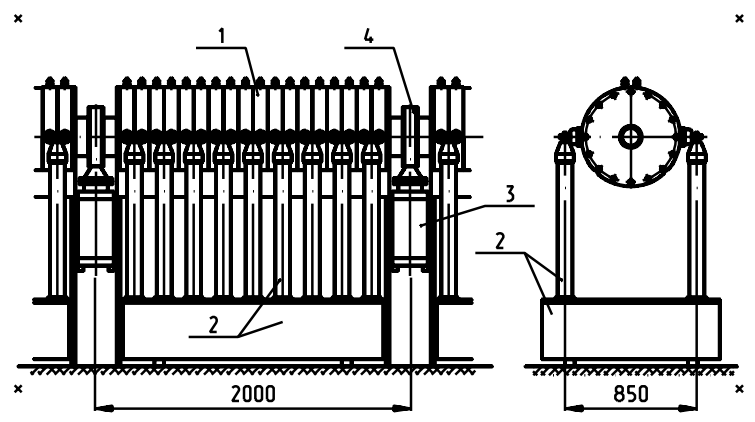

Figure. 1. Accelerating module. Here: (1) - induction section; (2) - modulator; (3) - sputter-ion pump.

\section{Accelerator design}

\section{A. Accelerating modules}

SILUND-21 accelerator consists of seven accelerating modules. Each module provides $1.5 \mathrm{MV}$ accelerating voltage at $1 \mathrm{kA}$ beam load. The general layout of the accelerating module is presented in Fig.1. Induction section (1) consists of 36 permalloy cores. The core exciting windings are commutated in parallel and the input impedance of the section is equal to $0.5 \Omega$ at nominal beam load. Pulses of accelerating voltage with $42 \mathrm{kV}$ amplitude and $50-70 \mathrm{~ns}$ duration are formed by modulator (2) with $0.5 \Omega$ internal resistance. TGI1-2500/50 hydrogen thyratron (2500 A, $50 \mathrm{kV}$ ), which is used as a commutator, does not provide commutation of the required level of the peak power, so nonlinear power compression schemes are used to increase the peak power.

The modulator scheme (see Fig.2) is based on the application of the nonlinear power compression technique. Its key element is nonlinear double forming line (NDFL) which consists of 18 double forming lines evenly spaced along the accelerating module. Hydrogen thyratron T operates in a pulsed mode with $3 \mu \mathrm{s}$ pulse duration and provides the following parameters: $U=30 \mathrm{kV}$ and $I=10 \mathrm{kA}$. Permalloy reactor $L$ and transformer $\operatorname{Tr}$ fulfil the roles of ferromagnetic switches. Transformer $T r$ is composed of three transformers with the transfer factor equal to $2 / 3$ which are placed along the accelerating module. NDFL is charged within the time period $1 \mu \mathrm{s}$ and then produces high-voltage pulse $(U=$ $42 \mathrm{kV}, I=84 \mathrm{kA}$, pulse duration $50-70 \mathrm{~ns}$, rise time about of $5 \mathrm{~ns})$.

The voltage of six inductors (4), commutated in a consecutive order, is fed to the accelerating gap (2) which is formed by cone diaphragm (1) (see Fig.3). The amplitude of accelerating voltage at each of six accelerating gaps of the accelerating section is equal to $250 \mathrm{kV}$.

Focusing of the electron beam is provided by guiding field of solenoid. The solenoid winding is sectional and the number of the sections is equal to the number of permalloy cores. Parameters

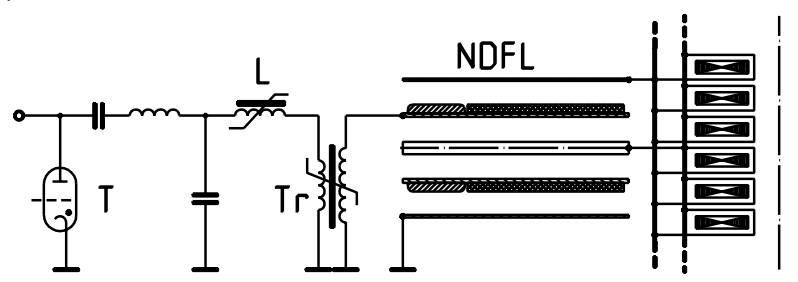

Figure. 2. Modulator scheme. Here: $\mathrm{T}-$ hydrogen thyratron; $\mathrm{L}$ - permalloy reactor; $\mathrm{Tr}$ - permalloy transformer; nonlinear double forming line. 


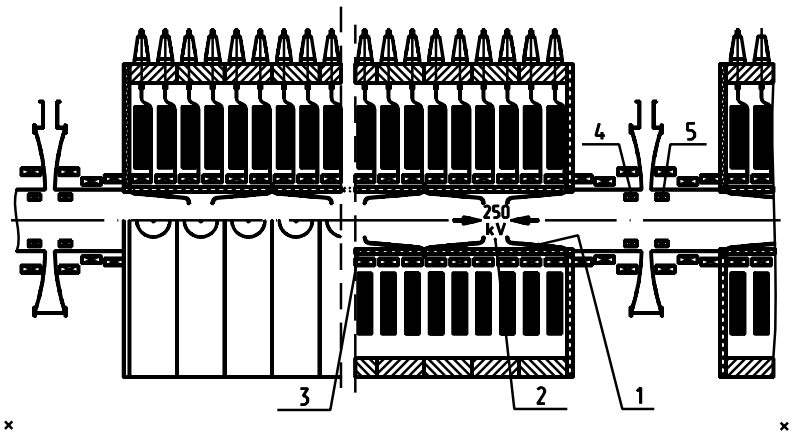

Figure. 3. Accelerating section. Here: (1) - diaphragm; (2) accelerating gap; (3) - magnetic lens; (4) - Rogowsky coil; (5) - beam position monitor.

of the solenoid power supply, developed for accelerator LUEK20 , allow one to provide the guiding magnetic field with the amplitude up to $1.4 \mathrm{~T}$ and pulse duration about of $0.8 \mathrm{~ms}$.

\section{B. Injection module}

The first accelerating module differs from all the other modules and is combined with the electron source. Accelerating voltage of the electron source is equal to $500 \mathrm{kV}-1 / 3$ of the total accelerating voltage of the module (see Fig.4) The choice of he electron source type depends on the mode of the accelerator operation. Our experience of work at the present accelerators has shown that at a low repetition rate (about of several cycles per second) it is more preferable to use the electron gun with graphite cathode (1) and gridded anode (2) [8](see Fig.4a). The value of magnetic field at the cathode is controlled by magnetic lens (3) to minimize the value of the electron beam emittance. At a high repetition rate (about of 50 cycles per second), we assume to use a plasma electron source without anode grid (see Fig.4b) [9], [?]. Ceramic tube (4) is destined to inject the plasma into the diode gap.

The both types of electron sources have demonstrated a high level of reliability and good output parameters during their operation at the accelerators SILUND, SILUND-II and SILUND-20.

\section{Electron beam parameters}

SILUND-21 will provide the electron beam with the following parameters: energy about of $10 \mathrm{MeV}$, peak current $\sim 1$ $\mathrm{kA}$ and pulse duration $\sim 50-70 \mathrm{~ns}$. Instant energy spread of electrons in the beam will be about of a fraction of per cent and the energy spread averaged over pulse duration will be $\sim 2-$ $3 \%$. At the accelerator exit we expect to obtain the values of the normalized emittance to be equal to $0.4 \pi \mathrm{cm} \cdot \mathrm{rad}, 0.15 \pi \mathrm{cm} \cdot \mathrm{rad}$ and $0.1 \pi \mathrm{cm} \cdot \mathrm{rad}$ at $90 \%, 70 \%$ and $50 \%$ of the nominal value of the beam current.

\section{Present status}

Three of the total number of seven accelerating modules are mounted in the site of the SILUND-21 accelerator. Now we (a)

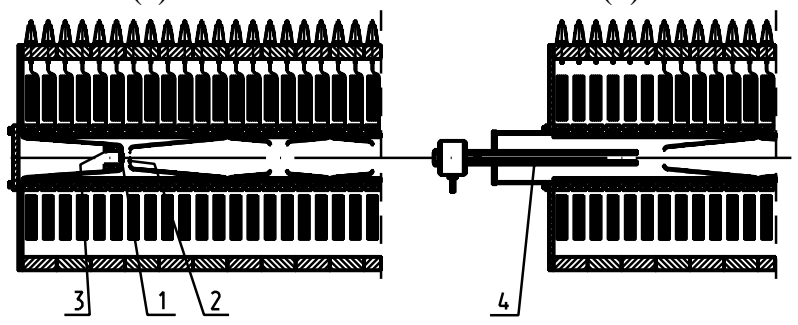

Figure. 4. Injection section. Here: (1) - graphite cathode; (2) gridded anode; (3) - magnetic lens; (4) - ceramic tube.

perform measurement of the electron beam parameters at the exit of the second accelerating module.

\section{References}

[1] G.V. Dolbilov et al., Proceedings of the EPAC94 Conference (London, 1994), V.1, p. 748

[2] B. Autin et al., preprint CERN/PS 94-16 (LP), CERN, Geneva, 1994

[3] V.S. Aleksandrov et al., Proceedings of the XIII International Conference on High Energy Accelerators", Novosibirsk, August 1986, vol.1, pp. 241-243

[4] G.V. Dolbilov et al., preprint JINR-9-83-307, Dubna, 1983

[5] B.G. Gorinov et al., preprint JINR-9-12148, Dubna, 1979

[6] L.S. Barabash et al., Proceedings IX International Conference on High Energy Accelerators. Stanford, USA, 1974, p. 318

[7] G.V. Dolbilov, V.A. Petrov, A.A. Fateev, preprint JINR-P986-290, Dubna, 1986

[8] G.V. Dolbilov et al., preprint JINR-9-89-154, Dubna, 1989

[9] G.V. Dolbilov et al., preprint JINR-P9-82-70, Dubna, 1982

[10] V.S. Aleksandrov et al., JINR publication P9-83-613, Dubna, 1983, p.54-60 